\title{
A CLINICAL STUDY OF CUTANEOUS MANIFESTATIONS IN PATIENTS WITH THYROID DISORDERS
}

\author{
A. Venkata Krishna ${ }^{1}$, K. Narasimha Prasad ${ }^{2}$, D. Subhash Reddy 3 , M. Sridevi ${ }^{4}$
}

${ }^{1}$ Associate Professor, Department of DVL, Osmania Medical College/Hospital.

${ }^{2}$ Assistant Professor, Department of DVL, Osmania Medical College/Hospital.

${ }^{3}$ Assistant Professor, Department of DVL, Osmania Medical College/Hospital.

${ }^{4}$ Junior Resident, Department of DVL, Osmania Medical College/Hospital.

\section{ABSTRACT}

\section{BACKGROUND}

The Thyroid Hormone (TH) is a key element in the endocrine control of epidermal development and function. Clinical evidence suggest that TH is involved in epidermal proliferation and differentiation, hair growth and wound healing besides affecting the function of dermal fibroblasts. Cutaneous signs of thyroid disease can be caused by the thyroid malady itself or be due to a primary cutaneous disease that has an associated risk of thyroid disease. Primary diseases of the skin, such as vitiligo or lichen sclerosus et atrophicus are associated with an increased prevalence of Autoimmune Thyroid Disease (AITD). This is a prospective crosssectional and comparative study carried out in the outpatient departments of Dermatology and Endocrinology at Osmania General Hospital during the period from November 2012 to August 2014 to study the cutaneous disorders associated with thyroid dysfunction.

\section{MATERIALS AND METHODS}

A total of 65 cases of who presented to OPDs of DVL and Endocrinology at OGH, who were previously diagnosed to have thyroid dysfunction were included in the study. Another 65 age and sex matched control group was taken who presented to the general OPD with non-thyroid disorders. The patients were examined clinically to look for the evidence of thyroid dysfunction manifesting in the skin and appendages in the form of textural and pigmentary changes and other associated conditions. Hair microscopy was done to rule out hair shaft disorders. Hair pull test was done to know the percentage of hairs in telogen and anagen. The skin findings in the patients and the control group were compared statistically. Chi square test was applied and $\mathrm{p}$ value was calculated. All the patients were subjected to laboratory investigations to evaluate the status of thyroid dysfunction. Thyroid profile (T3, T4 and TSH) was done in every case. Reference values for TSH (0.3 - $5.2 \mathrm{mIU} / \mathrm{L}), \mathrm{T} 4$ (5.0 - $12.5 \mu \mathrm{g} / \mathrm{dL})$, T3 (0.8 - $1.9 \mathrm{ng} / \mathrm{mL})$. Selected cases were further investigated by anti-TPO, Fine needle aspiration cytology and ultrasonography of the thyroid.

\section{RESULTS}

Dry coarse skin 20 (62.5\%), diffuse hair loss 17 (53.1\%), swelling of face and hands 10 (31.25\%), pruritus $9(28.1 \%)$ were the common symptoms among the hypothyroid group. In the hyperthyroid group swelling in the neck 13 (65\%), flushing 12 (60), pruritus $11(55 \%)$ and diffuse hair loss $8(40 \%)$ were the frequent symptoms. The percentage of symptoms reported were more in the study group in contrast to the controls. Among the associated skin disorders, chronic idiopathic urticaria, vitiligo, alopecia areata were observed with increased frequency in patients with thyroid disorders when compared to controls and had a statistically significant association $(p<0.05)$.

\section{CONCLUSION}

When compared for the presence of each dermatosis, pruritus, diffuse hair loss, hyperpigmentation, xerosis, flushing, goitre, hyperhidrosis, chronic urticaria, vitiligo, alopecia areata were found to be significantly higher in the patient group with thyroid diseases than in the control group. There is a convincing evidence of a significant association between thyroid autoimmunity and skin disorders like chronic idiopathic urticaria, vitiligo, alopecia areata. Assessing thyroid function should be considered standard practice in patients with a personal or family h/o any autoimmune diseases and also in patients presenting with chronic idiopathic urticaria, vitiligo, alopecia areata, telogen effluvium, polymorphic light eruption, diffuse or local hyperpigmentation, generalised pruritus, hyperhidrosis, xerosis and acquired palmoplantar keratoderma.

\section{KEYWORDS}

Hyperthyroidism, Hypothyroidism, Cutaneous Signs and Symptoms.

HOW TO CITE THIS ARTICLE: Krishna AV, Prasad KN, Reddy DS, et al. A clinical study of cutaneous manifestations in patients with thyroid disorders. J. Evolution Med. Dent. Sci. 2016;5(74):5489-5500, DOI: 10.14260/jemds/2016/1242

Financial or Other, Competing Interest: None.

Submission 09-08-2016, Peer Review 02-08-2016,

Acceptance 08-09-2016, Published 15-09-2016.

Corresponding Author:

Dr. A. Venkata Krishna,

H. No: 21-41, Vivekanandanagar, Dilsuknagar,

Hyderabad, Telangana-500060.

E-mail: drvkananthula@gmail.com

DOI: $10.14260 /$ jemds/2016/1242

\section{INTRODUCTION}

The Thyroid Hormone (TH) is a key element in the endocrine control of epidermal development and function. Clinical evidence suggest that $\mathrm{TH}$ is involved in epidermal proliferation and differentiation, hair growth and wound healing besides affecting the function of dermal fibroblasts. Cutaneous signs of thyroid disease can be caused by the thyroid malady itself or be due to a primary cutaneous disease that has an associated risk of thyroid disease. By binding to their receptors Thyroid Hormones (TH) regulate epidermal cell proliferation, differentiation and homeostasis. 
Several TH-responsive genes as well as genes critical for TH metabolism and action are expressed at epidermal level. The role of TH in skin is still controversial, although it is generally recognised that $\mathrm{TH}$ signalling is central for skin physiology and homeostasis. Thyroid hormone directly influences proteoglycan synthesis in the skin by stimulating fibroblasts. In addition, it plays a regulatory role in epidermal differentiation at least in part because of effects on keratinocytes. Finally, TH appears to be essential in hair formation and sebum production. Gross clinical manifestations of Thyroid Hormone (TH) imbalance are often first seen in the skin where $\mathrm{TH}$ plays an integral role in sustaining natural function. As a result, the skin presents important external markers associated with thyroid disease that can signal dermatologists to investigate and diagnose thyroid disorder.

\section{AIMS AND OBJECTIVES}

- To evaluate the cutaneous manifestations associated with hypo and hyperthyroid states.

- To study the other cutaneous disorders associated with thyroid dysfunction.

\section{CUTANEOUS MANIFESTATIONS OF THYROID DISEASE Specific Lesions}

- Thyroglossal duct cyst.

- Cutaneous metastases from thyroid malignancies.

\section{Nonspecific Lesions}

Hyperthyroidism (General)

- Alopecia.

- Plummer's nails.

- Textural alterations.

- Hyperpigmentation.

Graves' Disease

- Ophthalmopathy.

- Dermopathy (Pretibial Myxedema).

- Thyroid acropachy.

Hypothyroidism

- Congenital hypothyroidism.

- Generalised myxoedema.

- Textural alterations.

- Xerosis/keratoderma.

- Carotenemia.

- Alopecia.

- Brittle nails.

\section{Miscellaneous Disorders}

Thyroid disorders associated with other dermatologic or Systemic diseases

Immunologically mediated disorders.

- Alopecia areata.

- Anaemias.

- Bullous disorders.

- Pemphigus.

- Bullous pemphigoid.

- Herpes gestationis.

- Dermatitis herpetiformis.

Connective tissue diseases.

- Dermatomyositis.

- Lupus erythematosus.
- Scleroderma

- Endocrinopathies.

- G protein disorders.

- McCune-Albright syndrome.

II. Albright's hereditary osteodystrophy.

- Multiple endocrine neoplasia, types $2 \mathrm{a}$ and $2 \mathrm{~b}$.

- Acanthosis nigricans.

- Pustulosis palmoplantaris.

- Sweet syndrome.

- Urticaria/angioedema.

- Vitiligo.

- Miscellaneous disorders.

- Complications related to the treatment of thyroid disease.

\section{HYPOTHYROIDISM}

Iodine deficiency remains the most common cause of hypothyroidism worldwide. In areas of iodine sufficiency, autoimmune disease (Hashimoto's thyroiditis) and iatrogenic causes (Treatment of hyperthyroidism) are most common.

\begin{tabular}{|c|c|}
\hline Symptoms & Signs \\
\hline 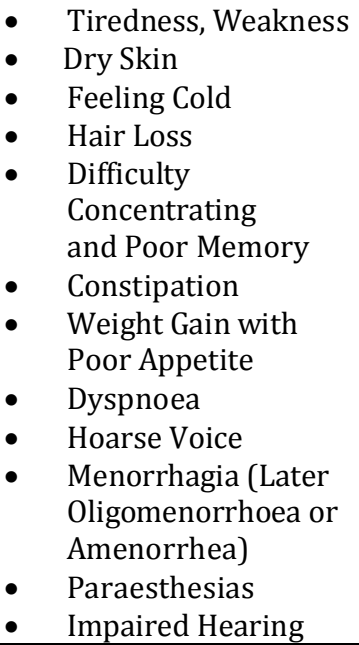 & $\begin{array}{ll}\text { - } & \text { Dry Coarse Skin; } \\
& \text { Cool peripheral } \\
& \text { Extremities } \\
\text { - } & \text { Puffy Face, Hands } \\
\text { and Feet } \\
\text { (Myxoedema) } \\
\text { Diffuse Alopecia } \\
\text { - } \quad \text { Bradycardia } \\
\text { - } & \text { Peripheral Oedema } \\
\text { - } & \text { Delayed Tendon } \\
& \text { Reflex Relaxation } \\
\text { (Woltman's Sign) } \\
\text { - } \quad \text { Carpal Tunnel } \\
\text { Syndrome } \\
\text { Serous Cavity } \\
\text { Effusions }\end{array}$ \\
\hline
\end{tabular}

\section{Skin}

- $\quad$ Pale, cold, scaly and wrinkled skin xerosis, asteatotic eczema, itch, palmoplantar keratoderma

- Absence of sweating

- Ivory-yellow skin colour

- Puffy oedema of hands, face and eyelids

- Purpura and ecchymosis

- Punctate telangiectasia on arms and fingertips

- Delayed wound healing

- Xanthomatosis (Secondary to hyperlipidaemia) Nails

- $\quad$ Brittle and striated nails

- Slow nail growth

- $\quad$ Coarse sparse scalp hair

- Loss of pubic, axillary and facial hair

- Loss of lateral eyebrows (Madarosis) Oral

- $\quad$ Large tongue

- Gingival swelling (congenital hypothyroidism)

- Oral candidiasis Cutaneous and Oral Features of Hypothyroidism 
Topical agents are available for the treatment of xerosis related to hypothyroidism including alpha-hydroxy acids, ointments with unctuous materials such as petrolatum or lanolin, liquid emulsions, oils and urea.

\section{THYROTOXICOSIS}

Thyrotoxicosis is defined as the state of thyroid hormone excess and is not synonymous with hyperthyroidism, which is the result of excessive thyroid function. However, the major aetiologies of thyrotoxicosis are hyperthyroidism caused by Graves' disease, toxic MNG and toxic adenomas. Hyperthyroidism is more commonly seen in women than men (5:1 ratio). Of these aetiologies, the most common cause of hyperthyroidism in young women is GD, whereas toxic nodular goitre is more common in older women.

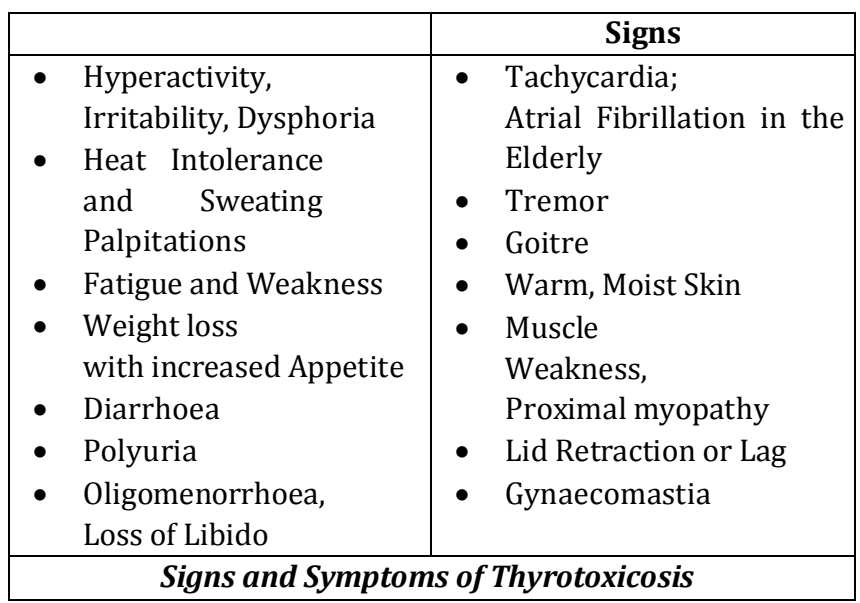

The skin changes of hyperthyroidism have been likened to infant's skin and described as soft, warm and velvety in texture. Warm skin is attributed to increased cutaneous blood flow and peripheral vasodilation. Skin moistness results from a combination of peripheral cutaneous vasodilation, increased sebaceous gland secretion and hyperhidrosis, which is most evident on palmar and plantar surfaces. Patients may also report facial flushing, which can be misconstrued for symptoms of rosacea. This flushing disorder results from increased blood flow through the skin.

\begin{tabular}{|ll|}
\hline \multicolumn{2}{|c|}{ Skin } \\
\hline$\bullet$ & Soft, Smooth, Velvety, Increased Skin Temperature \\
& Palmar Erythema, Facial Flushing, Increased Sweating \\
- & Pruritus \\
- & Hyperpigmentation \\
- & Othetibial Myxoedema \\
\hline & \multicolumn{2}{c|}{ Nails } \\
\hline$\bullet$ & Fast Nail Growth \\
- & Soft Nails \\
- & Koilonychias \\
- & Thistal Onycholysis \\
\hline & \multicolumn{1}{c|}{ Hair } \\
\hline$\bullet$ & Fine Thin Hair, Diffuse Alopecia \\
- & Alopecia Areata \\
\hline & $\quad$ Cutaneous Features of Hyperthyroidism \\
\hline
\end{tabular}

\section{Dermatologic Disorders Associated with Thyroid Disease $^{2}$}

Cutaneous signs of thyroid disease can be caused by the thyroid malady itself or be due to a primary cutaneous disease that has an associated risk of thyroid disease. Primary diseases of the skin, such as vitiligo or lichen sclerosus et atrophicus are associated with an increased prevalence of Autoimmune Thyroid Disease (AITD).

\section{Chronic Idiopathic Urticaria}

Subclinical thyroid autoimmunity and overt clinically important thyroid disease have been shown to occur with increased prevalence in patients with chronic urticaria. AITD and circulating anti-thyroid antibodies were notably elevated in patients with chronic urticaria and a positive ASST; 5\%$34 \%$ of patients with chronic urticaria have anti-thyroid antibodies despite being clinically or biochemically euthyroid and $5 \%$ and $10 \%$ of chronic urticaria patients have clinically apparent thyroid disease.

\section{Alopecia Areata}

The prevalence of thyroid autoimmunity ranged from $7 \%$ to $35 \%$ of patients with alopecia areata. Children with alopecia areata have an increased rate of a positive family history for thyroid disease.

\section{Vitiligo}

AITD follows the onset of vitiligo in both adult and paediatric populations. AITD is approximately three times more prevalent in adults with vitiligo than in the general population. Increased prevalence of AITD in children with vitiligo, anti-thyroid antibodies ranged from $12 \%$ to $24 \%$.

\section{Autoimmune Polyglandular Syndrome (APS)}

There is a well-known association among organ specific autoimmune conditions such as Graves' Disease (GD) and Hashimoto Thyroiditis (HT) and other organ-specific autoimmune diseases such as pernicious anaemia, myasthenia gravis, Immune Thrombocytopenia (ITP), type 1 diabetes mellitus and others. This constellation of diseases is sometimes referred to as Autoimmune Polyglandular Syndrome (APS).

\section{Connective Tissue Diseases}

Autoimmune thyroid disease, marked by the presence of antibodies directed against thyroid antigens has been associated with a number of non-organ-specific rheumatological disorders.

\section{Scleroderma}

$18 \%-52 \%$ of Systemic Sclerosis patients have been reported to have an anti-thyroid antibody. Higher prevalence of antithyroglobulin (Anti-TG) and anti-thyroid peroxidase (antiTPO) in patients with systemic sclerosis have been reported previously.

\section{Primary Sjögren's Syndrome}

Primary Sjogren's syndrome is perhaps the most frequent rheumatic autoimmune disease associated with AITD with a 10-fold higher prevalence compared with the general population. A strong association between primary Sjogren's syndrome and AITD points to a common pathogenic mechanism, in particular by the immunogenic predisposition and histology. 


\section{Autoimmune Blistering Disease}

AITD is reported more often in association with Pemphigoid gestationis than with Bullous pemphigoid. This may be due to the association of Human Leukocyte Antigens (HLA)-B8, DR3 and DR4 with both Pemphigoid gestationis and AITD, particularly Graves' disease. These associations support an autoimmune pathogenesis.

\section{Pemphigus Vulgaris}

Autoimmune disorders may accompany each other and coexistence of Pemphigus vulgaris with other autoimmune disorders such as myasthenia gravis, systemic lupus erythematosus, rheumatoid arthritis and Graves' disease has been reported.

\section{Dermatitis Herpetiformis}

Abnormal thyroid function tests have been found in 4\%-28\% of patients. Also, autothyroid antibodies occurred in $18 \%-$ $48 \%$ of Dermatitis herpetiformis patients. Hypothyroidism is slightly favoured over hyperthyroidism.

\section{Disorders of Pigmentation}

Melasma has a six-fold risk of AITD. Kiani et al evaluated the relationship of melasma and thyroid disorders. They found that there was a significant relationship between melasma and thyroid disorders, especially hypothyroidism and thyroid autoimmunity.

\section{Drug Effects on the Skin and Thyroid}

The Wolff-Chaikoff effect can occur with the use of Potassium Iodide (KI) or amiodarone also causes pigmentary effects on the skin. Bexarotene, used to treat Cutaneous T-cell lymphoma causes central hypothyroidism.

\section{Potassium Iodide}

The Wolff Chaikoff effect can occur, some may develop persistent hypo- or hyperthyroidism.

\section{Amiodarone \\ Amiodarone is an anti-arrhythmic drug. The main cutaneous toxicities of amiodarone include a drug exanthem, photosensitivity and hyperpigmentation. The discoloration is purple or blue-gray and is believed to be more common in patients with previous episodes of the photosensitivity. The effects on the thyroid include the Wolff-Chaikoff effect, hypothyroidism and hyperthyroidism.}

\section{Bexarotene}

Bexarotene is a rexinoid or retinoid X receptor (RXR) agonist. The most common side effects of bexarotene are hypertriglyceridemia and hypothyroidism, which are dose dependent. The mechanism is unclear; studies in rats have shown decreased levels of serum TSH soon after administration of an RXR agonist. Levels of T4 and T3 were slower to decline, hypothyroidism is monitored using free $\mathrm{T} 4$ levels, not TSH level which will be depressed due to bexarotene therapy.

\section{Drug Hypersensitivity Syndrome and Hypothyroidism}

Hypothyroidism has been reported to occur soon after or may be delayed by 3 or more months after an episode of Drug hypersensitivity syndrome. They have low levels of T4 and elevated levels of TSH and thyroid autoantibodies including antimicrosomal antibody.

\section{OTHER AUTOIMMUNE AND MISCELLANEOUS DISORDERS Addison's Disease}

The co-occurrence of Addison disease and AITD is known as APS type II or Schmidt syndrome. The triad of type 1 diabetes mellitus, AITD and Addison disease is known as Carpenter syndrome. The types of AITD diagnosed included HT, symptomless autoimmune disease, GD and isolated ophthalmopathy or myxoedema.

\section{Palmoplantar Pustulosis}

The prevalence of abnormal thyroid testing varies from $18 \%$ to $50 \%$. Anti-thyroid autoantibodies are less common and have been found in 14\%-25\% of patients with palmoplantar pustulosis. HT was much more common than GD in the same population.

\section{Acquired Palmoplantar Keratoderma}

Acquired palmoplantar keratoderma has been reported to occur secondary to hypothyroidism. A common and striking feature was the lack of response to topical corticosteroids but a total response to thyroid hormone replacement, mostly resolving in few weeks to months after hormone replacement.

\section{Acquired Ichthyosis}

The association of hypothyroidism and acquired ichthyosis may be due to the role of thyroid hormone in fatty acid metabolism and sterol production in the skin. Improvement of the ichthyosis with thyroid hormone replacement and recurrence with cessation of $\mathrm{T} 4$ administration.

\section{Lichen Sclerosus Et Atrophicus}

The prevalence of AITD was $12 \%$. The prevalence of GD was $6 \%-8 \%$ and HT was diagnosed in $4 \%-6 \%$ of LSA patients. Patients with LSA also had a high prevalence of autoantibodies (Anti-thyroid, antinuclear, anti-gastric parietal cell, among others), and commonly had a personal or family history of autoimmune disease $(60 \%)$.

\section{Scleromyxoedema}

Thyroid function testing is suggested as part of baseline laboratory studies, because of the clinical similarity to pretibial myxoedema. Thyroid function testing would also be important to distinguish the "dermato-neuro syndrome" of fever, seizures and coma associated with scleromyxoedema from thyrotoxicosis and pretibial myxoedema.

\section{Investigations and Screening for Thyroid Diseases}

The tests to detect thyroid dysfunction are:

1. Total thyroxine (TT4).

2. Free thyroxine (FT4).

3. Total triiodothyronine (TT3).

4. Free triiodothyronine (FT3).

5. Thyrotropin assay (TSH).

6. Radioactive iodine uptake assay.

7. Autoantibodies to thyroid peroxidase and thyroglobulin.

\section{PATIENTS AND METHODS}

This is a prospective cross-sectional and comparative study carried out in the outpatient departments of Dermatology 
and Endocrinology at Osmania General Hospital during the period November 2012 to August 2014.

A total of 65 cases of who presented to OPDs of Dermatology and Endocrinology at $\mathrm{OGH}$, who were previously diagnosed to have thyroid dysfunction were included in the study. Another 65 age and sex matched control group was taken who presented to the general OPD with non-thyroid disorders.

\section{Inclusion Criteria}

- Patients with clinical and biochemical evidence of thyroid dysfunction.

- $\quad$ Age 18-80 years.

- Willingness to participate in the study.

\section{Exclusion Criteria}

- Age less than 18 years.

- $\quad$ Patients who were acutely ill.

- Pregnant females.

- Patients who underwent surgical procedures for thyroid disease.

- Previous h/o radiation therapy to head and neck area.

Before enrolling for the study, the purpose and details of the study were explained to the patients in a language understood by him/her and informed consent was obtained.

After noting the demographic data, a detailed history of patient's presenting complaints and symptoms attributable to thyroid dysfunction was taken. The symptoms of hypothyroidism and hyperthyroidism were scored according to scoring of symptoms and signs of hypothyroidism and hyperthyroid symptom scale respectively. Detailed physical examination was done for each case, height and weight of each case was recorded. Tremor was examined on outstretched hands. Goitre when present was palpated for the presence of nodularity and was graded accordingly.

The patients were examined clinically to look for the evidence of thyroid dysfunction manifesting in the skin and appendages in the form of textural and pigmentary changes and other associated conditions. Hair microscopy was done to rule out hair shaft disorders. Hair pull test was done to know the percentage of hairs in telogen and anagen. The skin findings in the patients and the control group were compared statistically. Chi square test was applied and p value was calculated.

All the patients were subjected to laboratory investigations to evaluate the status of thyroid dysfunction. Thyroid profile (T3, T4 and TSH) was done in every case. Reference values for TSH (0.3-5.2 mIU/L), T4 (5.0-12.5 $\mu \mathrm{g} / \mathrm{dL})$, T3 $(0.8-1.9 \mathrm{ng} / \mathrm{mL})$. Selected cases were further investigated by anti-TPO, Fine needle aspiration cytology and Ultrasonography of the thyroid.

\section{RESULTS}

A total of 65 diagnosed cases of thyroid dysfunction who satisfied the inclusion and exclusion criteria attending the Dermatology and Endocrinology OPDs of Osmania General Hospital were taken up for the study to evaluate the cutaneous manifestations. A group of 65 cases who presented to the general OPD with non-thyroid disorders were taken as control. An informed consent was obtained. The patients were evaluated in detail with clinical examination and relevant investigations. The analysis of the results is as follows.

\begin{tabular}{|c|c|c|c|c|}
\hline $\begin{array}{c}\text { Age } \\
\text { (Yrs.) }\end{array}$ & $\begin{array}{c}\text { Hypothyroid \% } \\
(\mathrm{n}=32)\end{array}$ & $\begin{array}{c}\text { Hyperthyroid \% } \\
(n=20)\end{array}$ & $\begin{array}{c}\text { Others \% } \\
(n=13)\end{array}$ & $\begin{array}{c}\text { Control \% } \\
(\mathrm{n}=65)\end{array}$ \\
\hline $0-10$ & $0(0)$ & $0(0)$ & $0(0)$ & $0(0)$ \\
\hline $11-20$ & $1(3.1)$ & $1(5)$ & $2(15.4)$ & $3(4.6)$ \\
\hline $21-30$ & $2(6.3)$ & $12(60)$ & $7(53.9)$ & $19(29.2)$ \\
\hline $31-40$ & $11(34.4)$ & $5(25)$ & $1(7.7)$ & $16(24.6)$ \\
\hline $41-50$ & $9(28.1)$ & $1(5)$ & $2(15.4)$ & $12(18.5)$ \\
\hline $51-60$ & $3(9.4)$ & $0(0)$ & $0(0)$ & $10(15.4)$ \\
\hline $61-70$ & $4(12.5)$ & $1(5)$ & $1(7.7)$ & $5(7.7)$ \\
\hline $71-80$ & $2(6.3)$ & $0(0)$ & $0(0)$ & $0(0)$ \\
\hline
\end{tabular}

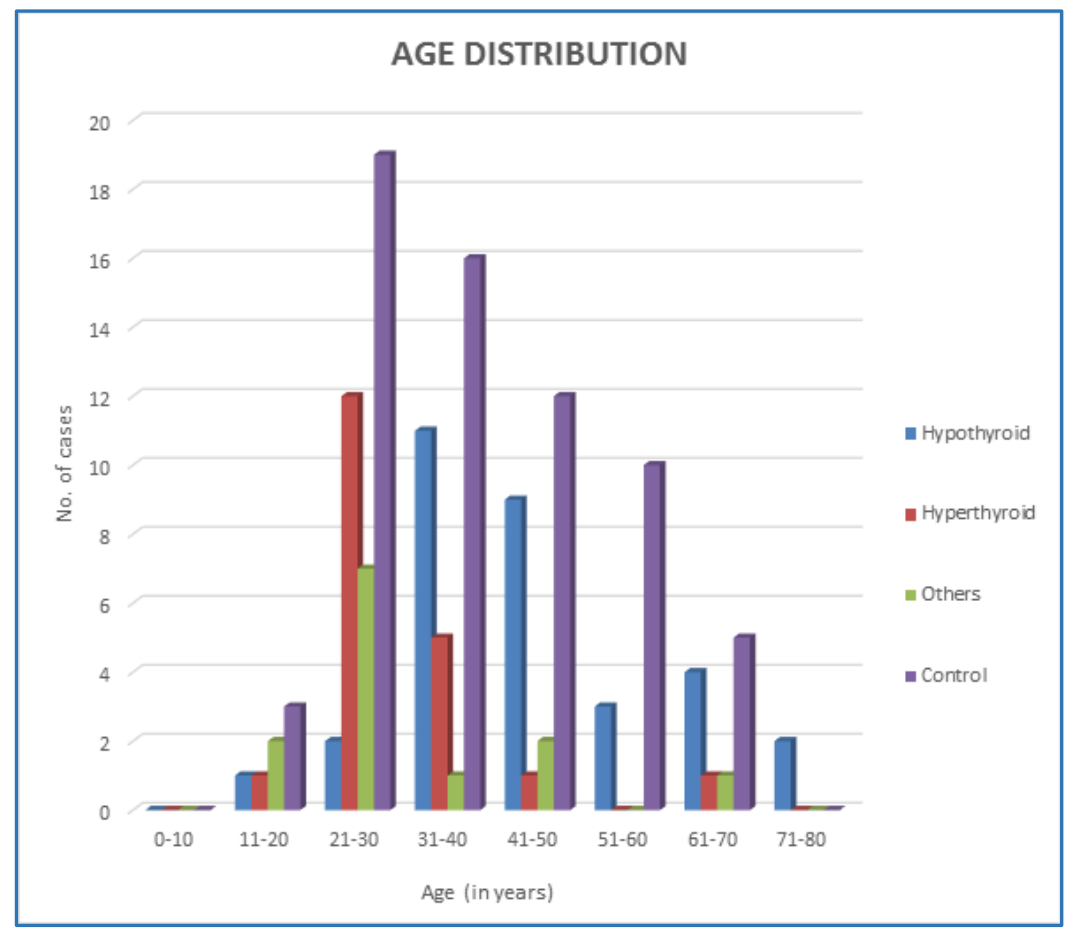


The age distribution in the study group ranges from 1880 years. In the hypothyroid, maximum patients were in the middle age group 31-50 yrs., 20 (62.5\%). In the hyperthyroid, large number of patients were in the age group 21-40 yrs., 17 (85\%). The age distribution of the control group ranged from 18-80 yrs. In the control group maximum number of people were in the age group of $21-40$ yrs., $52.8 \%$ of total control group.

\begin{tabular}{|c|c|c|}
\hline Condition & Male \% & Female \% \\
\hline Hypothyroid & $7(21.9)$ & $25(78.1)$ \\
\hline Hyperthyroid & $5(25)$ & $15(75)$ \\
\hline Others & $3(23.1)$ & $10(76.9)$ \\
\hline Controls & $30(46.2)$ & $35(53.8)$ \\
\hline \multicolumn{3}{|c|}{ Table 2: Sex Distribution } \\
\hline
\end{tabular}

Majority of the patients were females. Females accounted for 50 (76.9) out of total 65 cases. Male-to-female ratio is 1:3.33. In concordance to many previous studies, females had a higher incidence of thyroid dysfunction.
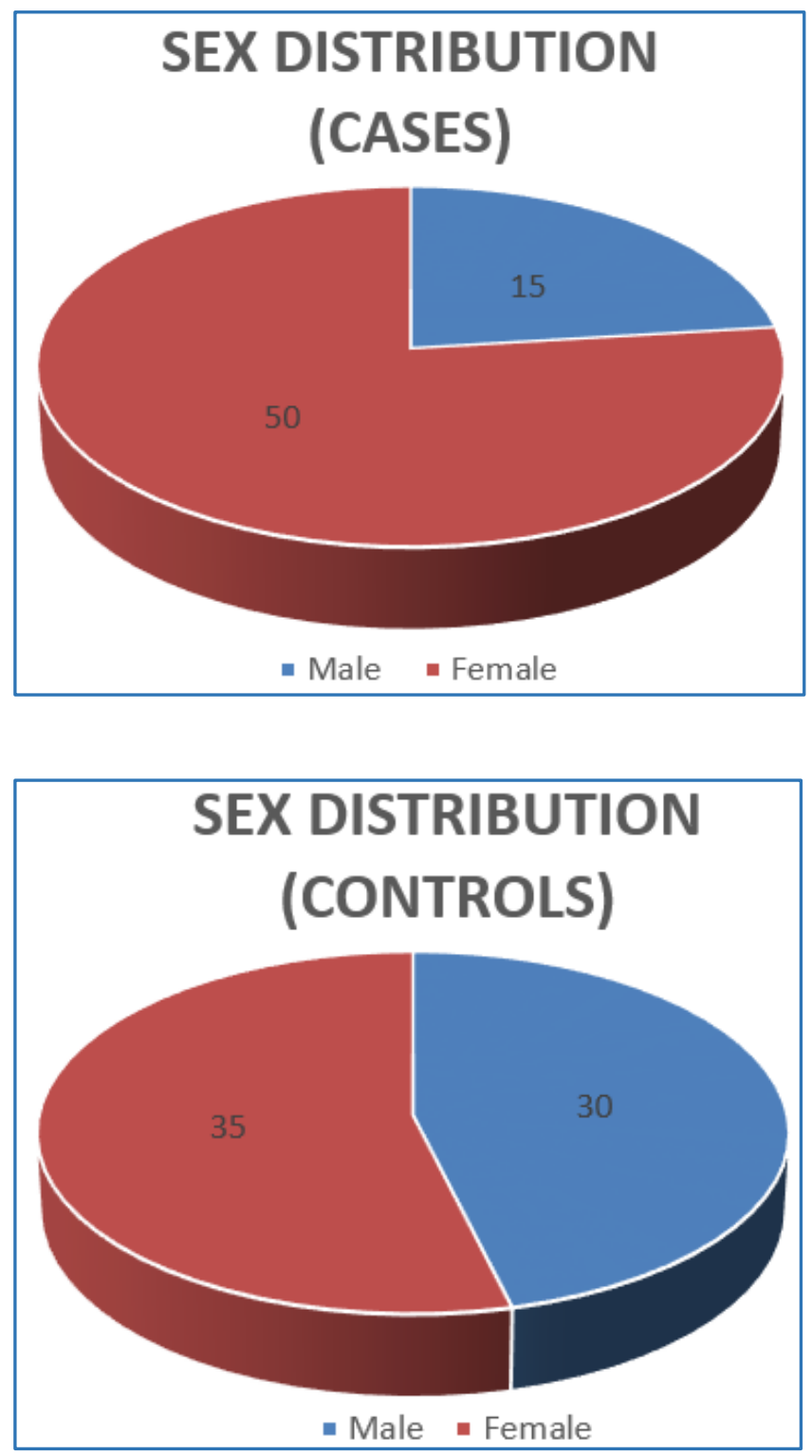

The predominant symptoms in patients with thyroid disorders include easy fatigability, weakness, weight gain, intolerance to cold, hoarseness of voice, nervousness, heat intolerance, increased sweating, hyperactivity, hyperdynamic precordium, tremor, loss of weight. These symptoms were significantly $(p<0.05)$ more in the study group than in the controls.

The most frequently observed findings on examination were diffuse loss of hair, xerosis, goitre, pale and cool extremities, puffiness of face, hyperhidrosis, warm moist skin and tremor. The less frequent findings include nail changes, madarosis, exophthalmos. Patients with thyroid dysfunction showed many cutaneous features, which are due to imbalance of circulating hormones, which were significantly more $(\mathrm{p}<0.05)$ than seen in control group.

Chronic idiopathic urticaria, acne, alopecia areata, acanthosis nigricans, vitiligo, tinea corporis, acrochordons, psoriasis, lichen planus, chronic lichenified eczema, keratosis pilaris, palmoplantar keratoderma, PLE were the frequent associated conditions seen in thyroid disorders group.

Among the associated skin disorders chronic idiopathic urticaria, vitiligo, alopecia areata were observed with increased frequency in patients with thyroid disorders when compared to controls and had a statistically significant association $(\mathrm{p}<0.05)$.

\begin{tabular}{|c|c|c|c|c|}
\hline Symptoms & $\begin{array}{c}\text { Hypothyroid } \\
\mathbf{\%} \\
\mathbf{( n = 3 2 )}\end{array}$ & $\begin{array}{c}\text { Hyperthyroid } \\
\mathbf{\%} \\
\mathbf{( n = 2 0 )}\end{array}$ & $\begin{array}{c}\mathbf{0 t h e r s} \\
\mathbf{\%} \\
(\mathbf{n = 1 3 )}\end{array}$ & $\begin{array}{c}\text { Controls } \\
\mathbf{\%} \\
(\mathbf{n = 6 5 )}\end{array}$ \\
\hline Pruritus & $9(28.1)$ & $11(55)$ & $4(30.8)$ & $5(10)$ \\
\hline $\begin{array}{c}\text { Hyperpig- } \\
\text { mentation }\end{array}$ & $6(18.7)$ & $4(20)$ & $3(23.1)$ & $4(8)$ \\
\hline Neck Swelling & $5(15.6)$ & $13(65)$ & $4(30.8)$ & $3(6)$ \\
\hline $\begin{array}{c}\text { Swelling of Face } \\
\text { and Hands }\end{array}$ & $10(31.25)$ & $0(0)$ & $1(7.6)$ & $0(0)$ \\
\hline Dry, Coarse Skin & $20(62.5)$ & $0(0)$ & $2(15.4)$ & $7(14)$ \\
\hline Hair Loss & $17(53.1)$ & $8(40)$ & $6(46.2)$ & $12(24)$ \\
\hline Flushing & $0(0)$ & $12(60)$ & $1(7.6)$ & $2(4)$ \\
\hline \multicolumn{5}{|c|}{ Table 3: Cutaneous symptoms } \\
\hline
\end{tabular}

Dry coarse skin 20(62.5\%), diffuse hair loss 17(53.1\%), swelling of face and hands $10(31.25 \%)$, pruritus $9(28.1 \%)$ were the common symptoms among the hypothyroid group.

In the hyperthyroid group swelling in the neck $13(65 \%)$, flushing $12(60)$, pruritus $11(55 \%)$, diffuse hair loss $8(40 \%)$ were the frequent symptoms. The percentage of symptoms reported were more in the study group in contrast to the controls.

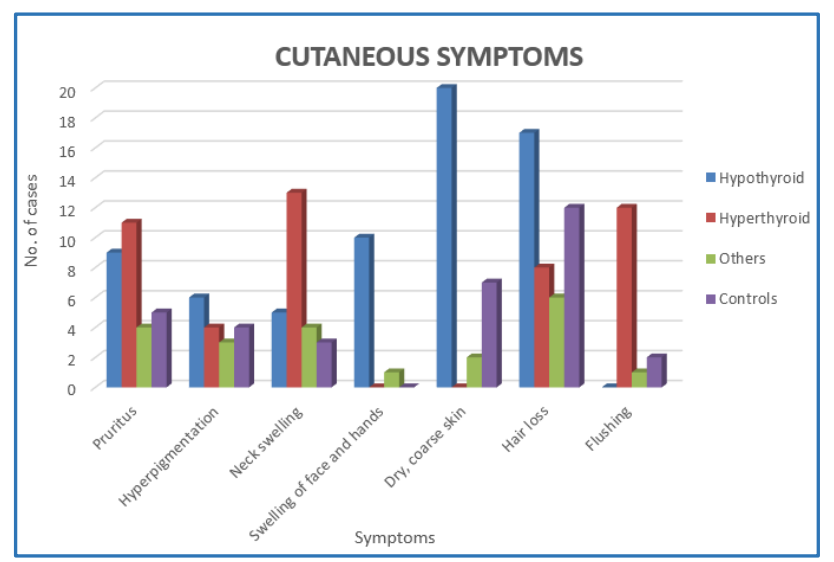




\begin{tabular}{|c|c|c|c|c|}
\hline Signs & $\begin{array}{c}\text { Hypothyroid \% } \\
(n=32)\end{array}$ & $\begin{array}{c}\text { Hyperthyroid \% } \\
(n=20)\end{array}$ & $\begin{array}{c}\text { Others \% } \\
(n=13)\end{array}$ & $\begin{array}{c}\text { Controls \% } \\
(n=65)\end{array}$ \\
\hline Goitre & $6(18.7)$ & $13(65)$ & $3(23.1)$ & $3(6)$ \\
\hline Cold Extremities & $17(53.1)$ & $0(0)$ & $1(7.7)$ & $1(2)$ \\
\hline Hyperpig-mentation & $5(15.6)$ & $4(20)$ & $4(30.8)$ & $3(6)$ \\
\hline Xerosis & $23(71.9)$ & $0(0)$ & $2(15.4)$ & $9(18)$ \\
\hline Puffiness of Face & $12(37.5)$ & $0(0)$ & $0(0)$ & $4(8)$ \\
\hline Hair Loss & $18(56.3)$ & $8(40)$ & $5(38.5)$ & $10(20)$ \\
\hline Madarosis & $5(15.6)$ & $0(0)$ & $0(0)$ & $0(0)$ \\
\hline Nail Changes & $6(18.7)$ & $3(15)$ & $1(7.7)$ & $4(8)$ \\
\hline Tremor & $0(0)$ & $12(60)$ & $0(0)$ & $2(4)$ \\
\hline Warm Moist Skin & $0(0)$ & $15(75)$ & $0(0)$ & $3(6)$ \\
\hline Hyperhidrosis & $0(0)$ & $14(70)$ & $2(15.4)$ & $2(4)$ \\
\hline Exophthalmos & $0(0)$ & $3(15)$ & $0(0)$ & $0(0)$ \\
\hline
\end{tabular}

In patients with hypothyroidism the most common findings on examination were xerosis 23 (71.9\%), diffuse hair loss 18 (56.3\%), cool and pale extremities 17 (53.1\%) followed by puffiness of face $12(37.5 \%)$, goitre $6(18.7 \%)$, madarosis $5(15.6 \%)$, nail changes $6(18.7 \%)$.
In the hyperthyroid group the examination findings were warm moist skin 15 (75\%), hyperhidrosis 14 (70\%), goitre 13 (65\%), tremor 12 (60\%), diffuse hair loss 8 (40\%), hyperpigmentation $4(20 \%)$, exophthalmos and nail changes in $3(15 \%)$ each.

All the findings observed were significantly higher in the thyroid dysfunction group when compared to the controls.

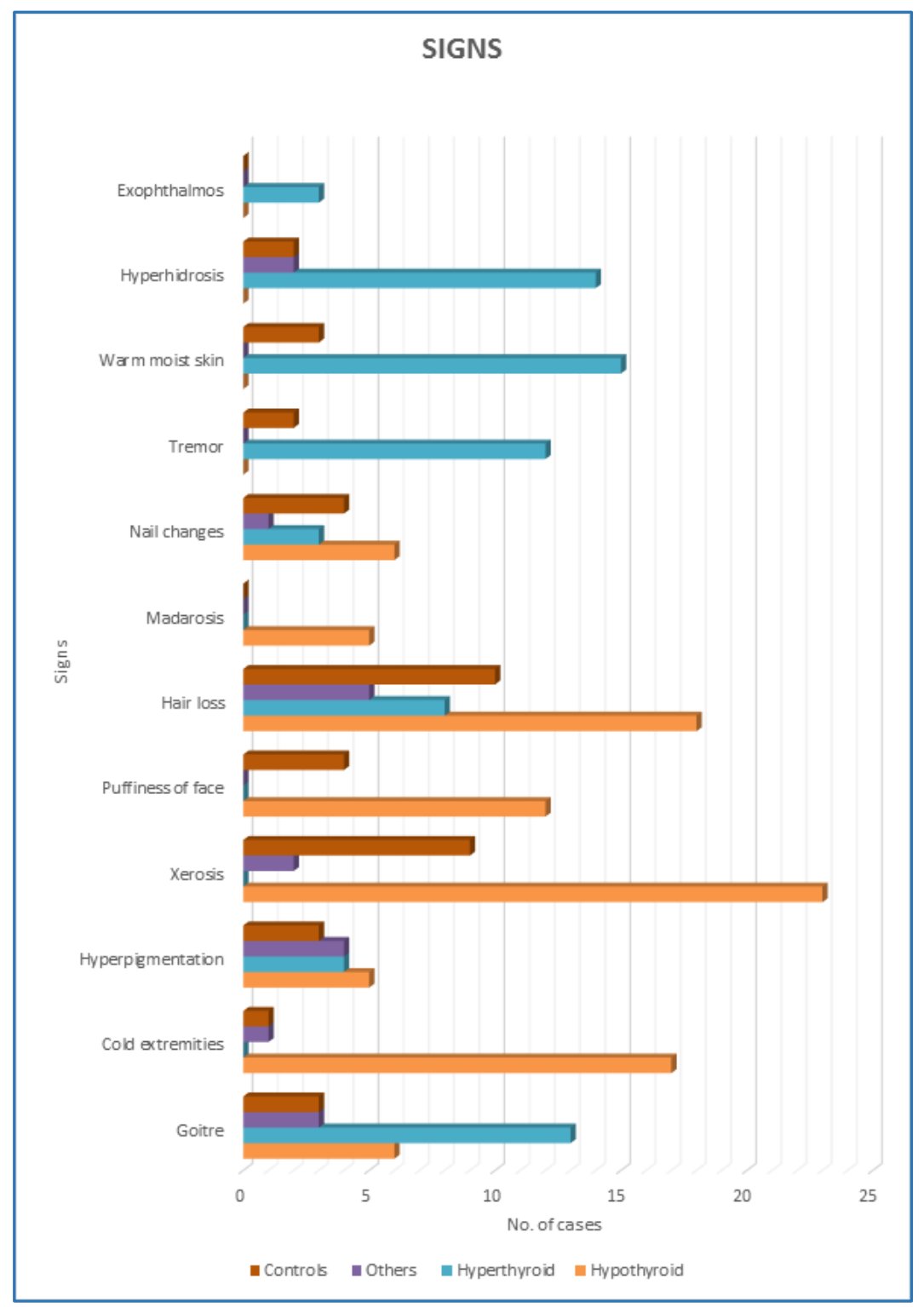




\begin{tabular}{|c|c|c|c|c|}
\hline Condition & $\begin{array}{c}\text { Hypothyroid \% } \\
(\mathrm{n}=32)\end{array}$ & $\begin{array}{c}\text { Hyperthyroid \% } \\
(\mathrm{n}=20)\end{array}$ & $\begin{array}{c}\text { Others \% } \\
(n=13)\end{array}$ & $\begin{array}{c}\text { Controls \% } \\
(n=50)\end{array}$ \\
\hline $\begin{array}{c}\text { Chronic Idiopathic } \\
\text { Urticaria }\end{array}$ & $7(21.8)$ & $3(15)$ & $0(0)$ & $0(0)$ \\
\hline Vitiligo & $3(9.4)$ & $1(5)$ & $1(7.7)$ & $0(0)$ \\
\hline $\begin{array}{l}\text { DLE with } \\
\text { Vitiligo }\end{array}$ & $1(3.1)$ & $0(0)$ & $0(0)$ & $0(0)$ \\
\hline Alopecia areata & $5(15.6)$ & $2(10)$ & $1(7.7)$ & $1(2)$ \\
\hline Bullous disorders & $0(0)$ & $0(0)$ & $0(0)$ & $0(0)$ \\
\hline Acne & $4(12.5)$ & $3(15)$ & $3(23.1)$ & $4(8)$ \\
\hline Acanthosis nigricans & $4(12.5)$ & $2(10)$ & $2(15.4)$ & $3(6)$ \\
\hline $\begin{array}{c}\text { Palmoplantar } \\
\text { keratoderma }\end{array}$ & $3(9.4)$ & $0(0)$ & $0(0)$ & $0(0)$ \\
\hline BT Hansen's & $1(3.1)$ & $0(0)$ & $0(0)$ & $0(0)$ \\
\hline Acrochordons & $2(6.3)$ & $0(0)$ & $2(15.4)$ & $0(0)$ \\
\hline $\begin{array}{l}\text { Chronic lichenified } \\
\text { eczema }\end{array}$ & $2(6.3)$ & $0(0)$ & $1(7.7)$ & $0(0)$ \\
\hline Tinea corporis & $2(6.3)$ & $3(15)$ & $0(0)$ & $0(0)$ \\
\hline $\begin{array}{c}\text { Granuloma } \\
\text { annulare }\end{array}$ & $1(3.1)$ & $0(0)$ & $0(0)$ & $0(0)$ \\
\hline Psoriasis & $2(6.3)$ & $1(5)$ & $0(0)$ & $0(0)$ \\
\hline PLE & $3(9.4)$ & $0(0)$ & $0(0)$ & $0(0)$ \\
\hline Keratosis Pilaris & $2(6.3)$ & $1(5)$ & $0(0)$ & $0(0)$ \\
\hline Lichen Planus & $1(3.1)$ & $2(10)$ & $0(0)$ & $0(0)$ \\
\hline LCV & $0(0)$ & $1(5)$ & $0(0)$ & $0(0)$ \\
\hline \multicolumn{5}{|c|}{ Table 5: Associated Skin Conditions } \\
\hline
\end{tabular}

When compared for the presence of each dermatosis, chronic idiopathic urticaria, vitiligo, alopecia areata, palmoplantar keratoderma, polymorphic light eruption were found to be significantly higher in patient's group with thyroid diseases than the control group.

\section{Clinical Pictures}

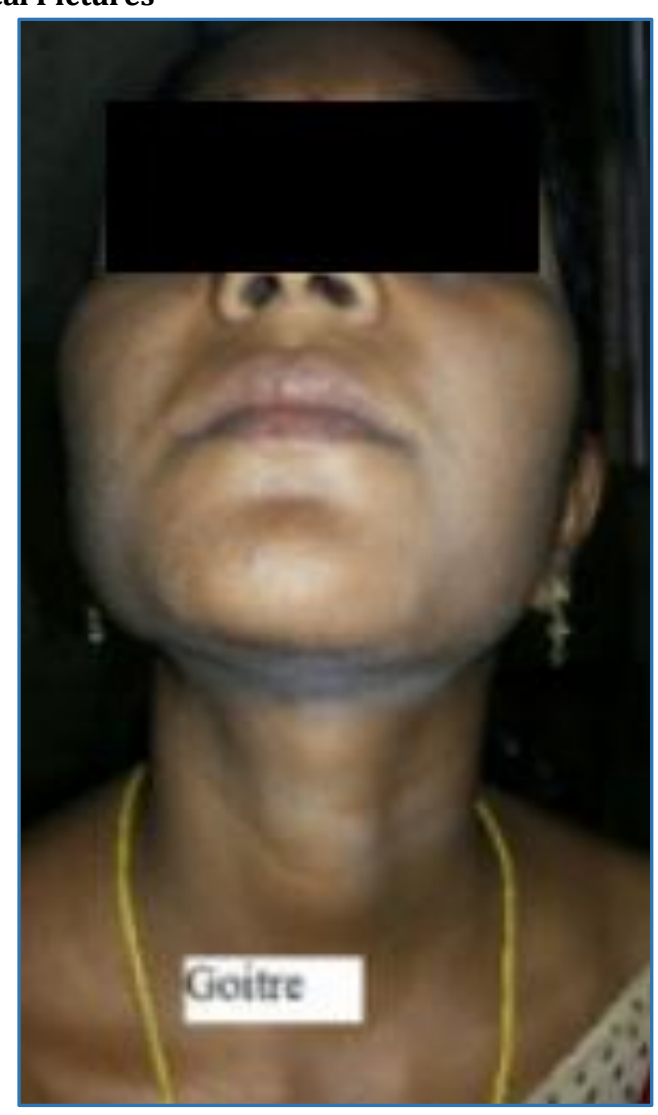

Goitre

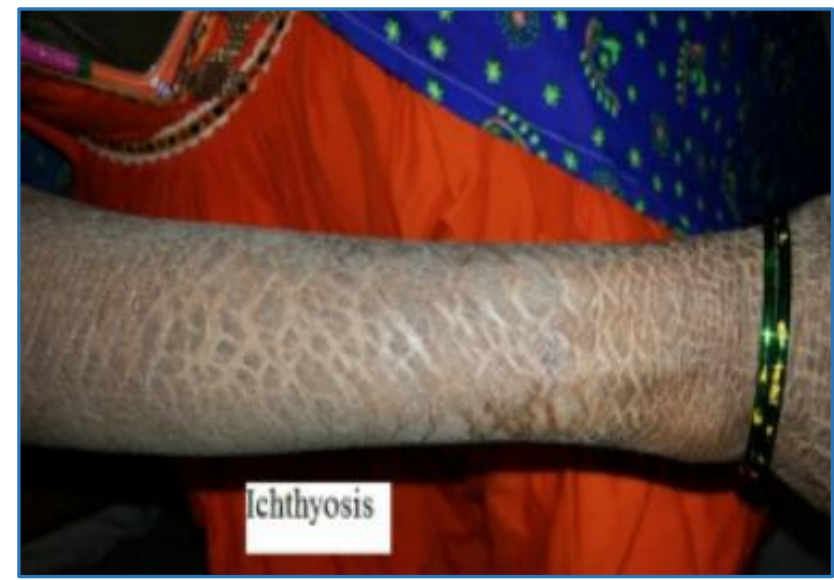

Ichthyosis

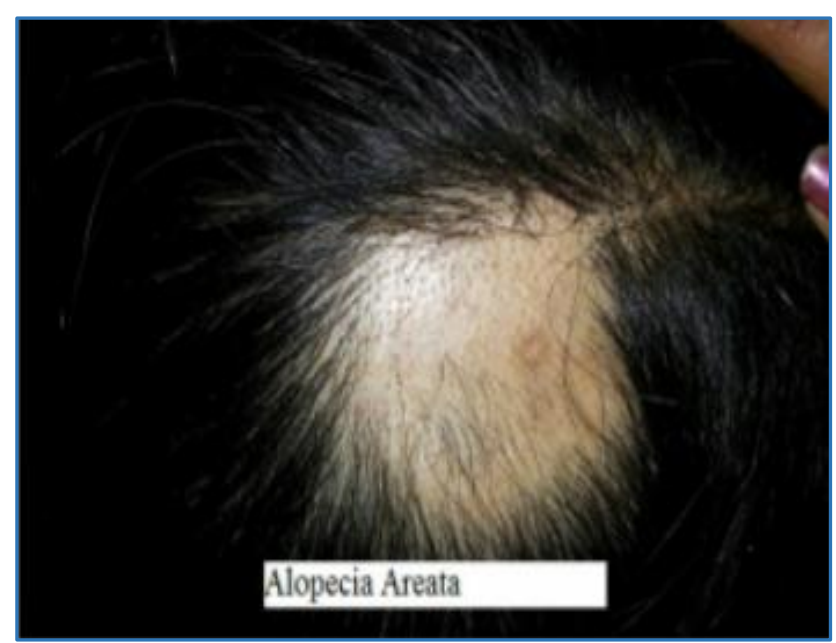

Alopecia Areata 


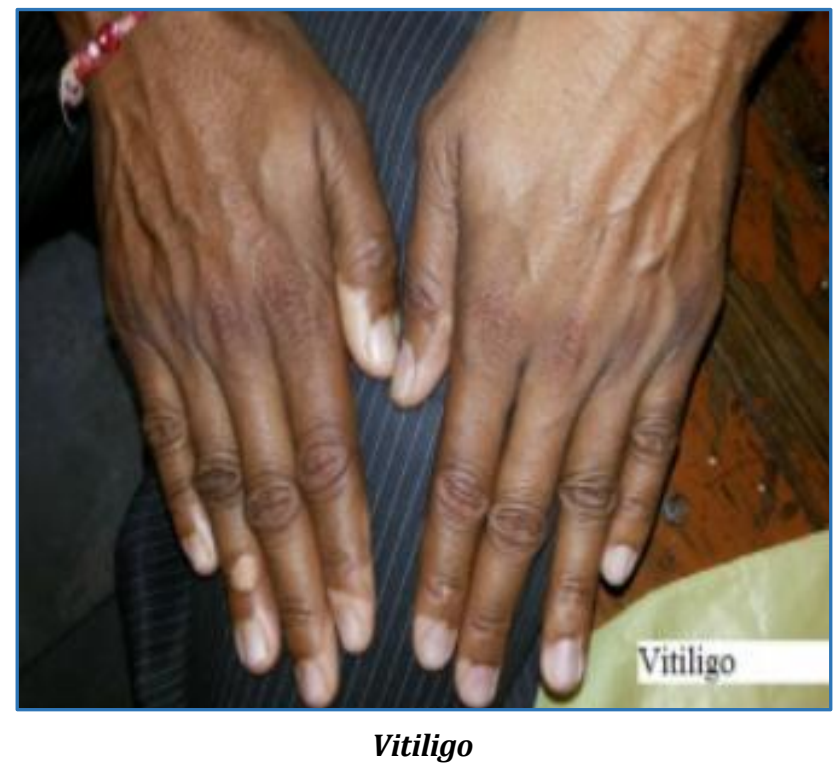

\section{DISCUSSION}

Thyroid hormones are instrumental in regulating the health and appearance of skin and when the thyroid gland becomes under or over functioning a variety of skin problems may result.

The diagnosis of thyroid disease can often first be identified by recognising various cutaneous manifestations associated with imbalance of circulating thyroid hormones. In order to ascertain this association of skin changes in relation to thyroid dysfunction, this study was carried out in the OPDs of Dermatology and Endocrinology, controls from general OPD at OGH from November 2012 to August 2014. A total of 65 patients were enrolled into the study who satisfied the inclusion criteria. An equal 65 age and sex matched controls who attended the general OPD with non-thyroid disorders were taken.

This discussion analyses of the data obtained from the study, compares it to that obtained in previous studies with similar objectives.

All the 65 cases enrolled into the study were already diagnosed cases of thyroid dysfunction and the 65 controls who attended the general OPD were not having thyroid related disorders. Out of the 65 cases, 32 (49.2\%) were hypothyroid and 20 (30.8\%) were hyperthyroid and 13 $(20 \%)$ cases had other thyroid disorders.

The age of the cases in the present study ranges from 1880 years. Maximum number of patients were in the age group 21-40 years who constituted $58.5 \%$ of the total study population. The age distribution of the control group ranged from 18-80 yrs. In the control group maximum number of people were in the age group of $21-40$ yrs., $52.8 \%$ of total control group. Mean age of the study group was 38.67 years, which corresponds to the study done by Khurram IM et al. ${ }^{1}$

In the study group of 65 patients, there were 50 (76.9\%) females and $15(23.1 \%)$ males, sex ratio, M:F was 1:3.33. In the control group, there were $35(53.8 \%)$ females and 30 $(46.15 \%)$ males. In a population based study conducted by Unnikrishnan AG et al, ${ }^{2}$ similar results were obtained suggesting increased prevalence of thyroid disorders in females. This observation of female preponderance may be due to increased association of autoimmune disorders in females, autoimmunity being an important cause of thyroid disorders.
In hypothyroid group the symptom complex is dominated by easy fatigability, weakness which were seen in 21 (65.6\%), $18(56.25 \%)$ respectively, a feature quite commonly seen in hypothyroidism. ${ }^{3}$ Easy fatigability and weakness were significantly more in hypothyroid patients than in controls 12 $(24 \%)$ and $10(20 \%)$ respectively.

Cold intolerance was noticed in 14 (43.8\%) cases, whereas in the controls it was noticed $3(6 \%)$ cases. Hypothermia is a result of hypometabolic state, which causes reduced core temperature and reflex vasoconstriction. ${ }^{4}$

Menstrual abnormalities in the study group were noted in $7(21.9 \%)$ patients as against $12 \%$ in the controls. Oligomenorrhoea and menorrhagia were the frequent symptoms. Menstrual abnormalities are an expected complication seen with thyroid disorders as discussed by Kakuno Y et al. 5

Weight gain has been reported by 15 (46.9\%) cases and 7 (14\%) of the control group. There is significant weight gain in patients with hypothyroidism as compared to controls. Similar observations were made by Dogra et al. ${ }^{6}$

The predominant cutaneous symptoms in the study group were dry coarse skin in $20(62.5 \%)$ followed by hair loss in 17 (53.1\%) swelling of face and hands in 10 (31.25\%). Pruritus was present in 9 (28.1\%) hyperpigmentation in 6 $(18.7 \%)$ and decreased sweating in 3 (4.61\%). Similar observations were made by Jabbour et $\mathrm{al}^{7}$ and Hueston et al ${ }^{8}$ in their studies. In the control group, the symptoms reported were pruritus $5(10 \%)$, hyperpigmentation 4 (8\%), neck swelling 3 (6\%), dry coarse skin 7 (14\%), hair loss 12 (24\%), flushing $2(4 \%)$. All the above reported symptoms were significantly higher in hypothyroidism than the controls.

The most common cutaneous sign observed was xerosis of skin in 23 (71.9\%), cold and pale extremities 17 (53.1\%), puffiness of face 12 (37.5\%), goitre in $6(18.7 \%)$ and hyperpigmentation in $5(15.6 \%)$ of cases. The examination findings in the control group include cool extremities $1(2 \%)$, hyperpigmentation $3(6 \%)$, xerosis $9(18 \%)$, puffiness of face $4(8 \%)$, loss of hair $10(20 \%)$, nail changes $4(8 \%)$. The above cutaneous findings were observed more commonly in patients with hypothyroidism than in controls. Xerosis 71.9\% is similar to that reported by Indra et al. ${ }^{9}$ Cool and pale extremities were seen in 17 (53.1\%) cases. Dry skin and cool peripheries can be explained by decreased cutaneous metabolism, reduced secretion of sweat and sebaceous glands, vasoconstriction and hyperkeratosis of stratum corneum.

Puffiness of face was seen in 12 (37.5\%), which was similar to that reported by Abid Keen et al. ${ }^{10}$

Melasma was noticed in $5(15.6 \%)$ cases of the study group. Hyperpigmentation in thyroid disorders has been reported mainly in hyperthyroidism. Significant association has been reported between thyroid autoimmunity and melasma, mainly in women whose condition developed during pregnancy or after ingestion of oral contraceptive pills. Thyroid dysfunction is one of the factors implicated in the pathogenesis of melasma apart from sunlight, genetic and other hormonal factors. ${ }^{11}$

The predominant finding on examination of hair was diffuse hair loss seen in $18(56.3 \%)$ in contrast to controls 10 (20\%). Dry coarse hair 10 (31.2\%) was seen. Similar observations were made by Rather PA et al.12 Hair loss is due to inhibition of initiation and duration of actively growing 
phase of hair cycle. Percentage of hairs in telogen are increased leading to telogen effluvium. As duration of anagen is affected, the rate of growth is slowed down in hypothyroidism. ${ }^{13}$

Nail changes were noticed in $6(18.7 \%)$, as against $4(8 \%)$ in the control group. The commonest among them was brittle nails $4(12.5 \%)$, longitudinal striations 2 (6.2\%), onycholysis $1(3.1 \%)$, pits 1 (3.1\%). Nail changes were less than that reported by N Puri. ${ }^{14}$

In patients with hyperthyroidism the presenting symptoms include weakness 16 (80\%), nervousness 15 (75\%), increased sweating 14 (70\%) followed by weight loss and hyperactivity $13(65 \%)$ each, intolerance to heat 12 (60\%), tremor in 11 (55\%), hyperdynamic pericardium 10 (50\%), diarrhoea 9 (45\%), shortness of breath 4 (20\%), oligomenorrhoea 6 (30\%), loss of libido 1 (5\%). In the controls, the symptoms include loss of weight $2(4 \%)$, increased sweating $6(12 \%)$, intolerance to heat $4(8 \%)$, nervousness $5(10 \%)$, weakness $10(20 \%)$, tremor $2(4 \%)$, hyperdynamic precordium 3 (6\%). Hyperthyroid group reported significantly more total symptoms than the controls. The association between the disease state and percentage of symptoms reported was statistically significant. The overall presentation is concordant with the clinical features described in literature. Boelaert et al ${ }^{15}$ reported the following symptoms in 3049 patients with hyperthyroidism. Weight loss $60.7 \%$, heat intolerance $54.9 \%$, tremor $53.9 \%$, palpitations $50.8 \%$ and anxiety in $41 \%$.

The mechanism by which the thyroid hormones can influence gastrointestinal motility, even if not still completely elucidated can be found in a synergism between a direct effect of the thyronins and an indirect effect mediated by catecholamines on the muscle cell receptors. Diarrhoea and malabsorption are common findings together with hyperthyroidism. 16

The cutaneous symptoms related to hyperthyroidism include the following - flushing in 12 (60\%), neck swelling 13 (65\%), pruritus 9 (28.1\%), hair loss 8 (40\%), hyperpigmentation in $4(20 \%)$. In the control group the symptoms include pruritus in $5(10 \%)$, neck swelling in 3 $(6 \%)$, dry coarse skin in $7(14 \%)$, loss of hair in $12(24 \%)$, flushing in 2 (4\%). The percentage of symptoms reported in the hyperthyroid group was significantly more than controls.

Most commonly observed cutaneous sign was warm moist skin 15 (75\%), hyperhidrosis $14(70 \%)$, goitre 13 $(65 \%)$, tremor $12(60 \%)$, hair loss $8(40 \%)$, pigmentation 4 (20\%), exophthalmos $3(15 \%)$, nail changes in $3(15 \%)$. These findings were less frequently found in the control group, warm moist skin 3 (6\%), tremor 2 (4\%), hyperhidrosis $2(4 \%)$, goitre $3(6 \%)$. The above findings matched with the study conducted by Trivalle et al 17 who observed that tachycardia, hyperhidrosis, heat intolerance, fatigue, nervousness, weight loss in more than fifty percent of patients aged less than 50 years.

In the present study warm, moist skin 15 (75\%), hyperhidrosis 14 (70\%) were similar to findings reported by $\mathrm{N}$ Puri et al. ${ }^{14}$ Hyperthyroidism is an hypermetabolic state resulting in imbalance between energy production and consumption leading to increased heat production and elimination. Thermogenesis leads to increased perspiration and heat intolerance as reported by Dabon CL et al. ${ }^{18}$

Warm skin, facial flushing occur as an effect of decreased peripheral vascular resistance, T3 inhibits the vascular smooth muscle contractility.

Hyperpigmentation was seen in 4 (20\%) of hyperthyroid group as against $6 \%$ in the controls. This hyperpigmentation is due to secondary release of pituitary ACTH compensating for accelerated cortisol degeneration as reported by $\mathrm{H}$. Niepomniszcze et al. ${ }^{19}$

On examination of hair, diffuse hair loss was seen in 8 $(40 \%)$ as against $20 \%$ of the controls. These clinical changes are due to alteration in the anagen/telogen ratio by excess thyroid hormones.

In the total study group, chronic urticaria 10 (15.4\%), vitiligo $5(7.7 \%)$, alopecia areata $8(12.3 \%)$ cases were the significant associations seen in patients with thyroid dysfunction. Similar associations have been reported earlier by S. Artantaş et al.20

In the controls $4(8 \%)$ had acne vulgaris, $3(6 \%)$ acanthosis nigricans and $1(2 \%)$ had alopecia areata. None of them from the control group had urticaria, vitiligo and other associated skin conditions seen in patients with thyroid dysfunction.

Thyroid autoimmunity is more prevalent in patients with chronic urticaria than in general population. Prevalence of positive thyroid autoantibodies ranged from 12 to $29 \%$ in patients with chronic urticaria in different studies. The mechanism linking thyroid autoimmunity and chronic urticaria are largely unknown. Thyroid antibodies do not induce urticaria and are only an epiphenomenon.

Alternate explanation for the increased incidence of thyroid disorders and chronic idiopathic urticaria is that these conditions reflect a shared genetic predisposition towards the development of autoimmune disease. It is found to occur frequently who express HLA-DR3, suggesting their aetiologies may involve common genetic factors Verneuil et al21 investigated the association between chronic urticaria and thyroid autoimmunity found higher frequency of thyroid autoantibodies in chronic urticaria.

The association between vitiligo and thyroid autoimmunity has been well-documented in literature. Betterle et $\mathrm{al}^{22}$ reported a study for establishing the relationship between vitiligo and thyroid and found a significant increase in thyroid autoimmunity in patients with vitiligo compared to normal, but no significant increase when compared with non-autoimmune controls. Alkhateeb et al 23 also found increased frequency of thyroid disease in patients with vitiligo.

In the study group, alopecia areata was seen in $8(12.3 \%)$ cases. It is frequently found to occur in autoimmune thyroid diseases. The prevalence of thyroid disorders in patients with alopecia areata ranges from 8-28\%. Lewinski and Broniarczyk-Dyla et al24 also confirmed the frequent coexistence of alopecia areata and thyroid abnormalities.

Palmoplantar keratoderma was seen in 3 (4.61\%) cases with hypothyroidism. It has been reported previously that it is due to longer mitotic division and decrease in epidermal steroidogenesis. Psoriasis was observed in 3 (4.61\%) cases of the entire study population. The exact role of thyroid hormones in the aetiopathogenesis of psoriasis is not known. Certain endocrinological disturbances exacerbate the disease. The skin is one of the target of thyroid hormones, T3 and T4 causes an increase in Epidermal Growth Factor (EGF) which leads to epidermal hyperplasia. Previous study conducted by 
Singh $\mathrm{S}$ et al25 showed the presence of autoantibodies including anti-TPO in patients with psoriasis and latent autoimmune diseases may develop in psoriasis patients without any clinical symptoms.

Three patients of the total study group (4.6\%) had polymorphic light eruption on examination. The probable relation between the two diseases is immune dysfunction causing hypersensitivity reaction in PLE and antibody generation in thyroid disease. Hasan et $\mathrm{al}^{26}$ stated that PLE is longstanding, slowly ameliorating disease with some tendency to development of autoimmune disease like thyroid disorders.

Borderline tuberculoid Hansen's was noted in 1 (1.54\%) patient with hypothyroidism. This patient did not develop any reaction during the follow-up period. Leprosy is less habitually associated with thyroid diseases. Few studies have reported decreased thyroid hormone levels with lepromatous reaction, lepromatous and borderline lepromatous states.

Leukocytoclastic vasculitis was present in 1 (1.54\%) patient with hyperthyroidism. As the patient is already on treatment for hyperthyroidism, the effect of anti-thyroid drugs cannot be ruled out.

Acne was seen in $6(15.4 \%)$ cases, whereas acanthosis nigricans 8 (12.3), acrochordons 4 (6.1\%), chronic lichenified eczema, lichen planus were noted in $3(4.6 \%)$ cases each. Tinea corporis was seen in $5(7.7 \%)$ cases. Keratosis pilaris in $3(4.6 \%)$ cases. Vitiligo with DLE was seen in one patient with hypothyroidism.

Granuloma annulare was seen in $1(1.54 \%)$ patient. The association of GA with thyroid disease has been described in the literature. The association of GA with autoimmune disorders, such as autoimmune thyroiditis supports the hypothesis that GA may be secondary to an immune attack. This association also has important therapeutic implications, it may be advantageous when treating patients with GA to consider checking a TSH and/or antimicrosomal or antithyroglobulin antibodies before administering medications, such as potassium iodide or interferon that could affect thyroid function.

\section{CONCLUSIONS}

- Skin presents an important external marker associated with thyroid diseases. These dermatological manifestations may occur secondary to the abnormal thyroid hormone levels or to the presence of thyroid autoantibodies that interact with the skin components. Sometimes, these signs and symptoms can be the presenting features of thyroid disorders.

- When compared for the presence of each dermatosis, pruritus, diffuse hair loss, hyperpigmentation, xerosis, flushing, goitre, hyperhidrosis, chronic urticaria, vitiligo, alopecia areata were found to be significantly higher in the patient group with thyroid diseases than in the control group.

- There is a convincing evidence of a significant association between thyroid autoimmunity and skin disorders like chronic idiopathic urticaria, vitiligo and alopecia areata.

- Assessing thyroid function should be considered standard practice in patients with a personal or family $\mathrm{h} / \mathrm{o}$ any autoimmune diseases and also in patients presenting with chronic idiopathic urticaria, vitiligo, alopecia areata, telogen effluvium, polymorphic light eruption, diffuse or local hyperpigmentation, generalised pruritus, hyperhidrosis, xerosis and acquired palmoplantar keratoderma.

- The key to diagnosing thyroid dysfunction from a dermatologic perspective is based on having a high index of suspicion that excess/deficient thyroid hormone is responsible for the patient's signs and symptoms.

- $\quad$ Equipped with the knowledge of the various cutaneous manifestations, dermatologists may be able to diagnose a potential thyroid disorder, which can be definitely established with routine thyroid function studies.

\section{REFERENCES}

1. Khurram IM, Choudhry KS, Muhammad K, et al. Clinical presentation of hypothyroidism: a case control analysis. J Ayub Medical College Abbotabad 2003;15(1):45-9.

2. Unnikrishnan AG, Menon UV. Thyroid disorders in India: an epidemiological perspective. Indian J Endocrinol Metab 2011;15(Suppl 2):S78-81.

3. Levy EG. Thyroid disease in the elderly. Med Clin North Am 1991;75(1):151-67.

4. Mullin GE, Eastern JS. Cutaneous signs of thyroid disease. Am Fam Physician 1986;34(4):93-8.

5. Kakuno Y, Amino N, Kanoh M, et al. Menstrual disturbances in various thyroid diseases. Endocr J 2010;57(12):1017-22.

6. Dogra A, Dua A, Singh P. Thyroid and skin. Indian J Dermatol 2006;51(2):96-9.

7. Jabbour SA, Miller JL. Review article: endocrinopathies and the skin. Int J Dermatol 2000;39(2):88-99.

8. Hueston WJ. Treatment of hypothyroidism. Am Fam Physician. 2001;64(10):1717-24.

9. Indra R, Patil SS, Joshi R, et al. Accuracy of physical examination in the diagnosis of hypothyroidism: a crosssectional, double-blind study. J Postgrad Med 2004;50(1):7-11.

10. Keen MA, Hassan I, Bhat MH. A clinical study of the cutaneous manifestations of hypothyroidism in Kashmir valley. Indian J Dermatol 2013;58(4):326.

11. Grimes PE. Melasma: etiologic and therapeutic considerations. Arch Dermatol 1995;131(12):1453-7.

12. Jamwal A, Sharma A, Rather PA. Cutaneous manifestations of hypothyroidism: prospective hospital based clinical study. J Adv Med Dent Sci 2013;1(2):5-12.

13. Freinkel RK, Freinkel N. Hair growth and alopecia in hypothyroidism. Arch Dermatol 1972;106(3):349-52.

14. Puri N. A study on cutaneous manifestations of thyroid disease. Indian J Dermatol 2012;57(3):247-8.

15. Boelaert K, Torlinska B, Holder RL, et al. Older subjects with hyperthyroidism patient with a paucity of symptomsand signs: a large cross sectional study. J Clin Endocrino Metab 2010;95(6):2715-26.

16. Pustorino S, Foti M, Calipari G, et al. Thyroid-intestinal motility interactions summary. Minerva Gastroenterol Dietol 2004;50(4):305-15.

17. Trivalle C. Hyperthyroidism in older individuals. J American Geriatric Society 1996;44(1):51-52.

18. Ojamaa K, Balkman C, Klein IL. Acute effects of triiodothyronine on arterial smooth muscle cells. Ann Thorac Surg 1993;56(Suppl 1):S61-S67. 


\section{Jemds.com}

19. Niepomniszcze H, Amad RH. Skin disorders and thyroid diseases. J Endocrinol Invest 2001;24(8):628-38.

20. Artantaş S, Gul U, Kilic A, et al. Skin findings in thyroid diseases. European Journal of Internal Med 2009;20(2):158-61.

21. Verneuil L, Leconte C, Ballet JJ, et al. Association between chronic urticaria and thyroid autoimmunity: a prospective study involving 99 patients. Dermatology 2004;208(2):98-103.

22. Betterle C, Callegari G, Presotto F, et al. Thyroid autoantibodies: a good marker for the study of symptomless autoimmune thyroiditis. Acta Endocr 1987;114:321-7.

\section{Original Research Article}

23. Alkhateeb A, Fain PR, Thody A, et al. Epidemiology of vitiligo and associated autoimmune diseases in Caucasian probands and their families. Pigment Cell Res 2003;16(3):208-14.

24. Lewinski A, Broniarczyk-Dyla G, Sewerynek E, et al. Abnormalities in structure and function of the thyroid gland in patients with alopecia areata. J Am Acad Dermatol 1990;23(4 Pt 1):768-9.

25. Singh S, Singh U, Singh S. Prevalance of auto antibodies in patients of psoriasis. J Clin Lab Anal 2010;24(1):44-8.

26. Hasan T, Ranki A, Jansen CT, et al. Disease association in polymorphous light eruption. A long term follow up study of 94 patients. Arch Dermatol 1998;134(9): 1081-5. 